\title{
Prediction of Interpolants in Zero Diluted Images
}

\author{
T. Kishan Rao, M. Shankar Lingam, Manish Prateek, and E. G. Rajan
}

\begin{abstract}
This paper provides an algorithmic procedure to predict interpolants of zero diluted images. Given a digital image, one can zero dilute it by right adjoining a column consisting of ' $0 s$ ' to every column except the last column and inserting a row consisting of ' 0 ' ' below every row except the last row. This yields a new image with a size $(2 \mathrm{~W}-1) \times(2 \mathrm{H}-1)$, where $W$ is the width and $H$ is the height of the original image. Another way of zero diluting an image is by right adjoining a column consisting of ' $0 \mathrm{~s}$ ' to every column and inserting a row consisting of ' 0 ' below every row. This yields a new image with a size $(2 W) \times(2 H)$, where $W$ is the width and $H$ is the height of the original image. Alternatively, subsampling of an image is carried out by forcing pixel values in the alternate columns and rows to zero. Thus, the size of the subsampled image is reduced to half of the size of the original image. This means $75 \%$ of the information in the original image is lost in the subsampled image. On the other hand, zero dilution of an image does not cause loss of information but increases the possibility of predicting more information. The question that arises here is whether it is possible to predict more pixel values, which are called interpolants so that the reconstructed image is an enhanced version of the original image in resolution. In this paper, two novel interpolant prediction techniques, which are reliable and computationally efficient, are discussed. They are (i) interpolant prediction using neighborhood pixel value averaging and (ii) interpolant prediction using extended morphological filtering. These techniques can be applied to predict interpolants in a subsampled image also.
\end{abstract}

Index Terms - Ground Penetrating Radar, Zero Diluted Imaging, Targeted Buried Object Detection, Interpolant Prediction.

\section{INTRODUCTION}

Digital zoom-in increases the size of the pixels leaving a pixelated image that often is not even suitable for viewing on screen. Resizing of an image also causes similar kind of pixilation problem. Alternatively, one can enlarge a given image using the technique described below.

\section{A. Concept of Zero Dilution}

Fig. 1 shows an array of size $9 \times 9$ and its zero diluted version of size $17 \times 17$. One can also zero dilute the $9 \times 9$ array by adding one column at the right and one more row at the bottom of the array so that one gets zero diluted array

Published on January 12, 2021.

T. Kishan Rao, Research Scholar, MG-NIRSA, University of Mysore, India.

(e-mail: kishanrao65@gmail.com)

Dr. M. Shankar Lingam, Research Associate, NIRDPR, Hyderabad, India.

(e-mail: shankumacharla@gmail.com)

Manish Prateek, Professor and Dean, University of Petroleum \& Energy

Studies, Dehradun, Uttarakhand, India.

(e-mail: mprateek@ddn.upes.ac.in)

E. G. Rajan, Adjunct Professor, Department of Cybernetics, University of Petroleum and Energy Studies, Dehradun, Hon. Director MG-NIRSA, NIRSA, Hyderabad, India.

(e-mail: dr.rajaneg@gmail.com) of size $18 \times 18$, which is the double the size of given array. Depending on requirement, one may choose either of the methods.

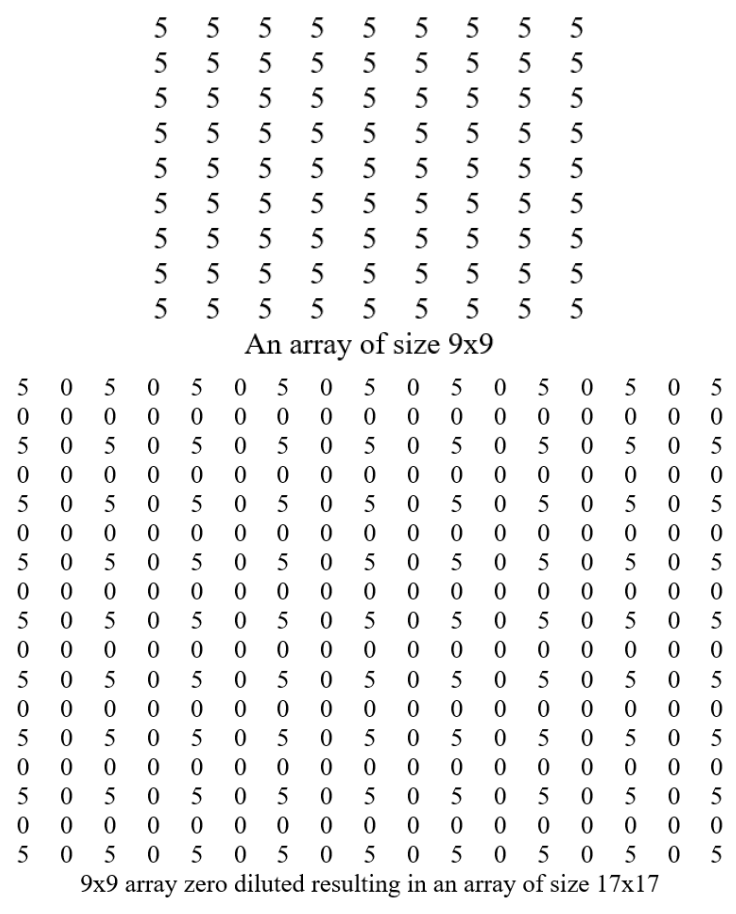

Fig. 1. An array of size 9x9 and its zero diluted form.

Fig. 2 shows a sample gold mine image of size $1177 \times 891$ and its zero diluted version of size $2353 \times 1781$. This is achieved using the technique demonstrated in Fig. 1. The 0 's are called the 'interpolants'. The question that arises here is whether it is possible to predict suitable values for the interpolants so that the enlarged image does not show up pixilation effects. In this paper, two techniques of (i) interpolant prediction using neighborhood pixel value averaging and (ii) interpolant prediction using extended morphological filtering are briefly explained. Consider the sample gold mine image of size $1177 \times 891$ shown in Fig. 1 . After applying the zero dilution algorithm to this image, one gets the zero diluted image of size $2353 \times 1781$, which is also shown in Fig. 1.

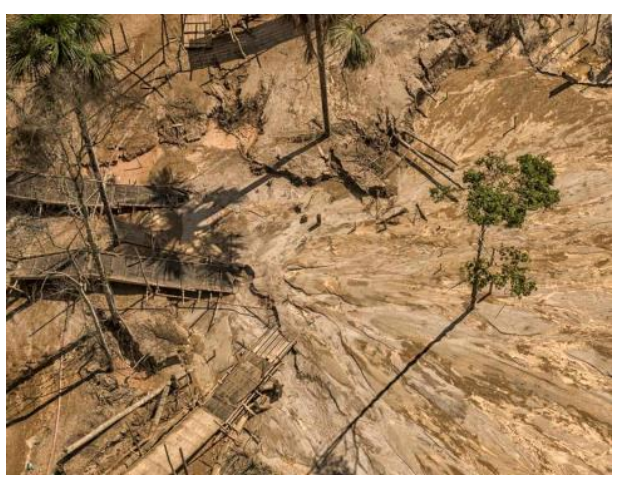

Gold mine image $($ Size $=1177 \times 891)$ 


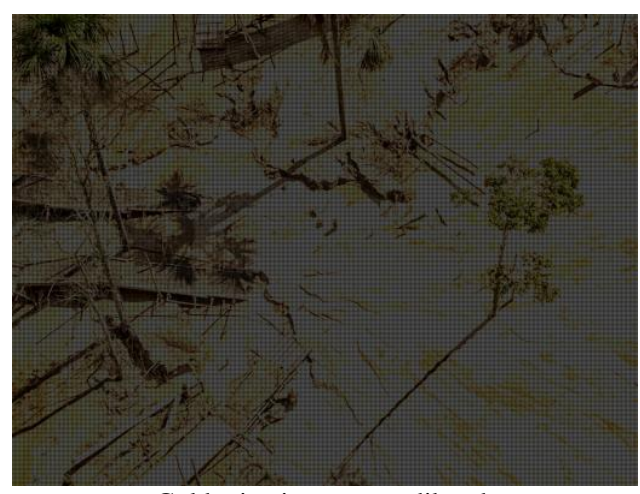

Gold mine image zero diluted.

$($ Size $=2353 \times 1781)$

Fig. 2. A sample gold mine image and its zero diluted form.

In what follows, the image reconstruction is carried out using the two interpolant predicting techniques mentioned above.

\section{INTERPOLANT PREDICTION TECHNIQUES}

\section{A. Interpolant Prediction Using Neighborhood Pixel Value} Averaging

This is a computationally intensive process for interpolant prediction.

\section{\begin{tabular}{|l|l|l|}
\hline$i-1, j-1$ & $i-1, j$ & $i-1, j+1$ \\
\hline
\end{tabular}

\begin{tabular}{|l|l|l|}
\hline$i, j-1$ & $i, j$ & $i, j+1$ \\
\hline
\end{tabular} \\ \begin{tabular}{l|l|l|}
$i+1, j-1$ & $i+1, j$ & $i+1, j+1$
\end{tabular}}

9-neighborhood window

\begin{tabular}{l|l|l}
\hline$i-1, j-1$ & & $i-1, j+1$ \\
\hline & $i, j$ & \\
\hline$i+1, j-1$ & & $i+1, j+1$ \\
\hline
\end{tabular}

5-neighborhood window 2
Scan the subsampled image with the 9-neighborhood window shown in Fig. 3. At every position, the interpolant is evaluated as the average of all available non-zero values using the prediction formula.

$\{x(i-1, j-1)+x(i-1, j)+x(i-1, j+1)+x(i, j-1)+x(i, j+1)+x(i+1, j-1)+x(i+1, j)+x(i+1, j+1)\}$

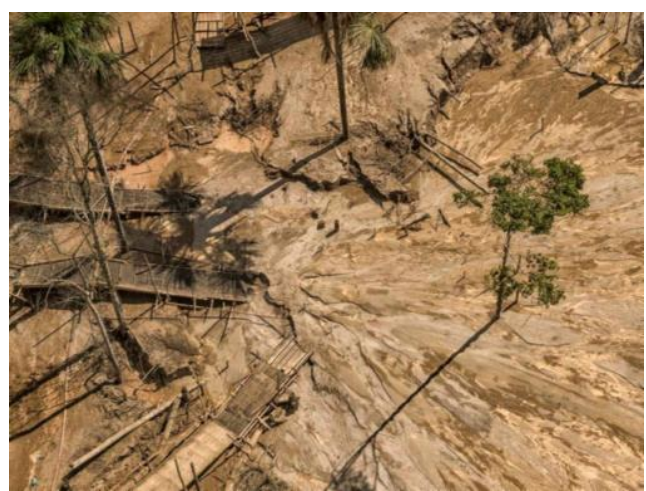

Reconstructed image using pixel averaging. $($ Size $=2353 \times 1781)$

Fig. 4. A sample gold mine image zero diluted and its pixel averaged version.

\section{B. Interpolant Prediction Using Extended Morphological Filtering}

Morphological filtering makes use of two fundamental operations of 'dilation' and 'erosion', which are defined as follows. Dilation: Let image to be dilated be A and the structuring element that dilates $\mathrm{A}$ be $\mathrm{B}$. Then the dilation of $\mathrm{A}$ by $\mathrm{B}$ is defined as the Minkowski addition $\mathrm{D}(\mathrm{A}, \mathrm{B})=$ $\mathrm{A} \oplus \mathrm{B}=\operatorname{EXTSUP}_{(\mathrm{x}, \mathrm{y}) \in \mathrm{D}_{\mathrm{B}}}\left[\mathrm{A}_{\mathrm{x}, \mathrm{y}}+\mathrm{B}(\mathrm{x}, \mathrm{y})\right]$, where $\mathrm{D}_{\mathrm{B}}$ is the domain of the image $\mathrm{B}$, and EXTSUP is an operation of supremum over the union of the domains. Erosion: Let image to be eroded be $\mathrm{A}$ and the structuring element that erodes $\mathrm{A}$ be $\mathrm{B}$. Then the erosion of $\mathrm{A}$ by $\mathrm{B}$ is defined as the Minkowski subtraction $\mathrm{A} \theta \mathrm{B}=\mathrm{INF}_{(\mathrm{x}, \mathrm{y}) \in \mathrm{D}_{\mathrm{B}}}\left[\mathrm{A}_{\mathrm{x}, \mathrm{y}}+\mathrm{B}(\mathrm{x}, \mathrm{y})\right]$ as $\mathrm{E}(\mathrm{A}, \mathrm{B})=\operatorname{INF}_{(\mathrm{x}, \mathrm{y}) \in \mathrm{D}_{\mathrm{B}}}\left[\mathrm{A}_{-\mathrm{x}, \mathrm{y}}-\mathrm{B}(\mathrm{x}, \mathrm{y})\right]$, where $\mathrm{D}_{\mathrm{B}}$ is the domain of the image $\mathrm{B}$, and INF is an operation of infimum over the intersection of the domains. Following these definitions, morphological filtering operations of Closing and Opening are defined in the following manner. Closing of A by B is represented as $A o B$ and defined as $A o B=(A \oplus B) \theta B$. Opening of $\mathrm{A}$ by $\mathrm{B}$ is represented as $\mathrm{A} \bullet \mathrm{B}$ and defined as $\mathrm{A} \bullet \mathrm{B}=(\mathrm{A} \theta \mathrm{B}) \oplus \mathrm{B}$. Same structuring element should be used for dilation and erosion in any morphological filtering. Now, $(\mathrm{AoB}) \mathrm{O}(\mathrm{AoB})=\mathrm{AoB}$ and $(\mathrm{A} \bullet \mathrm{B}) \bullet(\mathrm{A} \bullet \mathrm{B})=\mathrm{A} \bullet \mathrm{B}$ which means closing and opening are idempotent operators.

\section{Extended morphological filters}

These are essentially morphological filters except that the structuring element need not remain the same for dilation and erosion in any morphological filtering. With reference to Fig. 5, for example, one can use the structuring element E11337799 for dilation and subsequently E1379 for erosion, so that extended closing operation is carried out on a given image. The structuring elements of E11337799 and E1379 are shown in the respective dialog boxes. Extended morphological filtering is a computationally less intensive process for interpolant prediction. At every position, the interpolant is evaluated using the procedure given below. Scan the given subsampled image and dilate it with E11337799 shown in Fig. 5. Subsequently, erode with E1379 shown in Fig. 5. The resulting image is extended morphological filtered version, which is also the interpolant predicted version. By extended morphological filtering, one can reconstruct the original image from the subsampled version, which means the lost information is predicted. Let us take some sample images and do the process of 
subsampling and extended morphological filtering. Fig. 8 shows a sample image of a gold mine, its subsampled version, the subsampled image dilated using structuring element E-11337799 and the dilated image eroded again using structuring element E-1379. Scan subsampled image with E-11337799. At every position, find the maximum image pixel value and assign it to the central pixel and thus dilation is carried out. Then, scan dilated version of subsampled image with E-1379. At every position, find the minimum image pixel value and assign it to the central pixel and thus erosion is carried out.

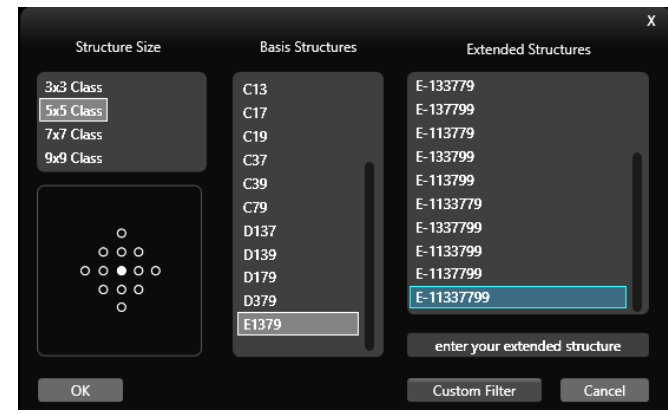

Neighborhood structure E-11337799

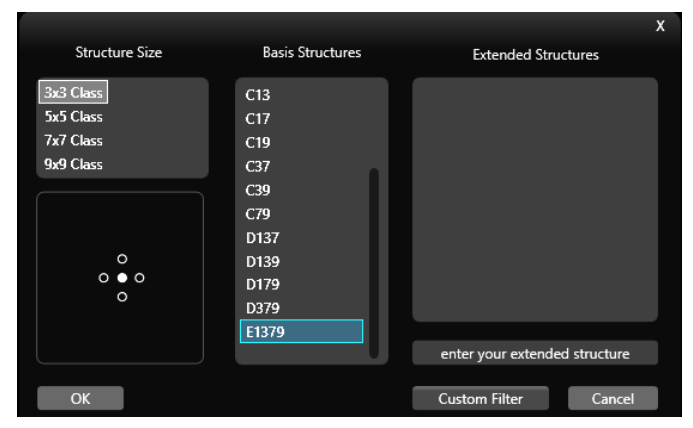

Neighborhood structure E-1379

Fig. 5. Structuring elements used for extended morphological filtering.

\section{Information gain due to zero dilution and} reconstruction using extended morphological filtering

Given an image of size $100 \times 100$, size of its zero diluted version turns out to be $199 \times 199$. The number of pixels in the zero diluted version is 39,601 , whereas number of pixels in the original image is 10,000 . This means $396.01 \%$ additional predicted information is added to the original image by zero diluting it followed by extended morphological filtering.

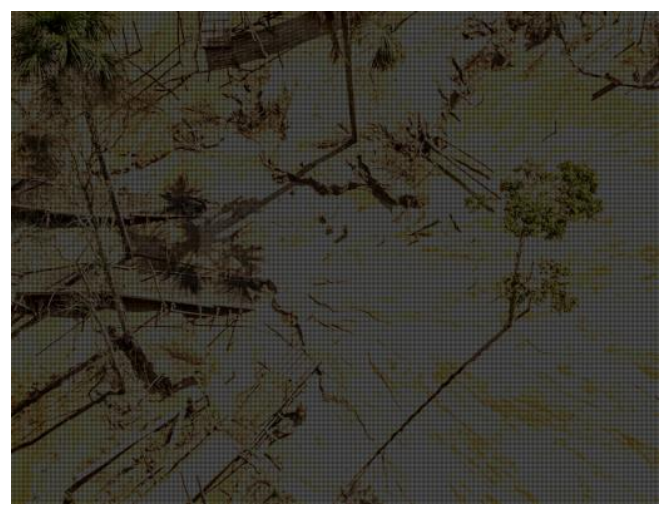

Gold mine image zero diluted. $($ Size $=2353 \times 1781)$

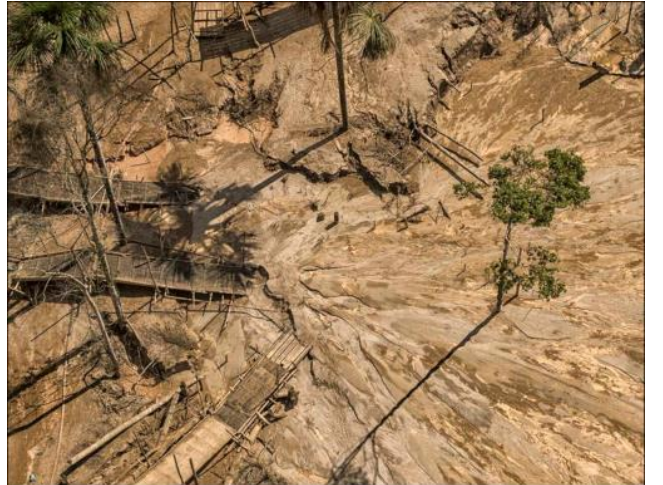

Reconstructed image using extended morphological filter. $($ Size $=2353 \times 1781)$

Fig. 6. A sample gold mine image zero diluted and its extended morphological filtered version.

3. Comparative study of prediction using neighborhood pixel value averaging and extended morphological filtering

A comparative study is made on the two interpolant prediction techniques in terms of visual quality analysis. Visual quality parameters include Trade of Threshold (ToT), entropy and visual quality. 'Statistical parameters' is also another measure by which one can compare the two techniques. However, due to space restriction, this paper provides results of comparative study based on visual quality parameters only.

C. Visual Quality Parameters of Gold Mine Image, Its Subsampled Image, and Reconstructed Images Using Prediction Formula and Extended Morphological Filter

Visual quality increases as one decreases the entropy of an image. The entropy of an image refers to the degree of randomness in the given image. Noise adds to entropy but reduces visual quality and filtering reduces entropy but increases visual quality. For better visual quality and considerable entropy, one has to accept a trade-off between the two. Visual quality is generally improved by quantizing an image with a threshold value (Th). The number of segments in an image depends on threshold value (Th). More the threshold value, less the number of segmented regions in an image. Less the number of segments, more is the visual quality. The visual quality parametric values of the original gold mine image, its zero diluted and the reconstructed images using extended morphological filter are presented in Table 1.

TABLE I: Visual Quality Parameters OF The ORIGINAL Gold Mine IMAGE, ITS ZERO DILUTED IMAGE AND THE RECONSTRUCTED IMAGES Using PIXEL AVERAGING AND EXTENDED MORPHOLOGICAL FILTERING METHODS

\begin{tabular}{cccccc}
\hline & \multicolumn{2}{c}{$\begin{array}{c}\text { Gold mine image } \\
(\text { Size }=1177 \times 891)\end{array}$} & \multicolumn{3}{c}{$\begin{array}{c}\text { Gold mine image } \\
\text { zero diluted } \\
\text { Size }=2353 \times 1781)\end{array}$} \\
\hline Th & $\begin{array}{c}\text { Visual } \\
\text { Quality }\end{array}$ & Entropy & Th & $\begin{array}{c}\text { Visual } \\
\text { Quality }\end{array}$ & Entropy \\
\hline 0 & 0 & 100 & 0 & 0.004390682 & 99.99560932 \\
\hline \hline 1 & 0 & 100 & 1 & 0.004390682 & 99.99560932 \\
\hline 2 & 0.001239622 & 99.99876038 & 2 & 0.004390682 & 99.99560932 \\
\hline 3 & 0.008200575 & 99.99179943 & 3 & 0.017371828 & 99.98262817 \\
\hline 4 & 0.029464855 & 99.97053514 & 4 & 0.017371828 & 99.98262817 \\
\hline 5 & 0.072756261 & 99.92724374 & 5 & 0.017371828 & 99.98262817 \\
\hline 6 & 0.177838042 & 99.82216196 & 6 & 0.041496717 & 99.95850328 \\
\hline 7 & 0.327164785 & 99.67283522 & 7 & 0.041496717 & 99.95850328 \\
\hline 8 & 0.586150374 & 99.41384963 & 8 & 0.041496717 & 99.95850328 \\
\hline \hline 9 & 0.957274053 & 99.04272595 & 9 & 0.081060579 & 99.91893942 \\
\hline 10 & 1.440345111 & 98.55965489 & 10 & 0.081060579 & 99.91893942 \\
\hline
\end{tabular}

Vol 5 | Issue 1 | January 2021 


\begin{tabular}{|c|c|c|c|c|c|c|c|c|c|c|c|}
\hline 11 & .053862518 & 97.94613748 & 11 & 0.081060579 & 99.91893942 & 91 & 89.08408164 & 10.91591836 & 91 & 14.72073951 & 85.27926049 \\
\hline 12 & 2.805359362 & 97.19464064 & 12 & 0.125325334 & 99.87467467 & 92 & 89.46931793 & 10.53068207 & 92 & 15.2112789 & 84.7887211 \\
\hline 13 & 3.682439423 & 96.31756058 & 13 & 0.125325334 & 99.87467467 & 93 & 89.83119213 & 10.16880787 & 93 & 15.27551648 & 84.72448352 \\
\hline 14 & 4.649249028 & 95.35075097 & 14 & 0.125325334 & 99.87467467 & 94 & 90.18858461 & 9.811415391 & 94 & 15.54792966 & 84.45207034 \\
\hline 15 & 5.770629928 & 94.22937007 & 15 & 0.215835424 & 99.78416458 & 95 & 90.5391115 & 9.460888504 & 95 & 15.82136892 & 84.17863108 \\
\hline 16 & 7.00348143 & 92.99651857 & 16 & 0.215835424 & 99.78416458 & 96 & 90.87018586 & 9.129814143 & 96 & 16.09845913 & 83.90154087 \\
\hline 17 & 8.373358812 & 91.62664119 & 17 & 0.33949039 & 99.66050961 & 97 & 91.20545586 & 8.794544139 & 97 & 16.36729295 & 83.63270705 \\
\hline 18 & 9.775180293 & 90.22481971 & 18 & 0.33949039 & 99.66050961 & 98 & 91.52 & 8.47405424 & 98 & 16.64070835 & \\
\hline 19 & 11.28952129 & 88.71047871 & 19 & 0.395495447 & 99.60450455 & 99 & 91.82889024 & 8.171109757 & 99 & 16.91803718 & 83.08196282 \\
\hline 20 & 12.85478213 & 87.14521787 & 20 & 0.395495447 & 99.60450455 & 100 & 92.13107188 & 7.868928118 & 100 & 17.2900043 & 82.7099957 \\
\hline 21 & 14.48984321 & 85.51015679 & 21 & 0.514735868 & 99.48526413 & 101 & 92.41685237 & 7.583147628 & 101 & 17.45544233 & 82.54455767 \\
\hline 22 & 16.13958904 & 83.86041096 & 22 & 0.514735868 & 99.48526413 & 102 & 92.70015362 & 7.299846382 & 102 & 17.73405974 & 94026 \\
\hline 23 & 17.83548694 & 82.16451306 & 23 & 0.645835904 & 99.3541641 & 103 & 92.97353789 & 7.02646211 & 103 & 18.01134085 & 65915 \\
\hline 24 & 19.53281517 & 80.46718483 & 24 & 0.645835904 & 99.3541641 & 104 & 93.23395381 & 6.766046188 & 104 & 18.29547046 & 52954 \\
\hline 25 & 21.27229054 & 78.7 & 25 & 0.73 & 99.26728109 & 105 & & 4578 & 105 & 306013 & 3987 \\
\hline 26 & 23.0218736 & 76.9781264 & 26 & 0.912856179 & 99.08714382 & 106 & 93.74906432 & 6.250935676 & 106 & 18.86418308 & 81692 \\
\hline 27 & 24.78490179 & 75.21509821 & 27 & 0.912856179 & 99.08714382 & 107 & 93.98325748 & 6.016742522 & 107 & 19.16141316 & 80.83858684 \\
\hline 28 & 26.49453088 & 73.50546912 & 28 & 1.008448961 & 98.99155104 & 108 & 94.22851187 & 5.771488128 & 108 & 19.46017043 & 82957 \\
\hline 29 & 28.23267128 & 71.76732872 & 29 & 1.213 & 98.78666846 & 109 & 94.4. & 5.545 & 109 & 25289 & 74711 \\
\hline 30 & 29.98625927 & 70.01374073 & 30 & 1.213331542 & 98.78666846 & 110 & 94.66323768 & 5.336762318 & 110 & 20.0502399 & 77601 \\
\hline 31 & 31.70494714 & 68.29505286 & 31 & 1.328300594 & 98.67169941 & 111 & 94.87874115 & 5.121258845 & 111 & 20.35248108 & 79.64751892 \\
\hline 32 & 33.45414878 & 66.54585122 & 32 & 1.445417262 & 98.55458274 & 112 & 95.07555495 & 45055 & 112 & 438819 & 61181 \\
\hline 33 & 35.0942637 & 64.9057363 & 33 & 1.57 & 98.42916195 & 113 & 95.27475262 & 77376 & 113 & 1583 & 8417 \\
\hline 34 & 36.7910198 & 63.2 & 34 & 1.696258829 & 98.30374117 & 114 & 95.46040982 & 90181 & 1114 & 1118 & 68882 \\
\hline 35 & 38.39613925 & 61.60386075 & 35 & 1.696258829 & 98.30374117 & 115 & 95.63929677 & 4.360703228 & 115 & 21.57841197 & 158803 \\
\hline 36 & 40.02280904 & 59.97719096 & 36 & 1.96 & 98.03376196 & 116 & 95.82180724 & 92765 & 116 & 81854 & 8146 \\
\hline 37 & 41.66 & 58.3390785 & 37 & 2.11 & 97.8 & 117 & 95.94 & 2786 & 117 & 51424 & 8576 \\
\hline 38 & 43.17316467 & 56.82683533 & 38 & 2.27232107 & 97.72767893 & 118 & 96.15869828 & 01717 & 118 & 22.53958951 & 41049 \\
\hline 39 & 44.70 & 55.29962134 & 39 & 2.42 & 1438 & 119 & 66333 & 667 & 119 & 19033 & 0967 \\
\hline 40 & 46.2 & 53.7 & 40 & 2.58 & 088 & 120 & 96. & 3.5 & 120 & 3438 & 1562 \\
\hline 41 & 47.67537549 & 52.32462451 & 41 & 2.743126256 & 97.25687374 & 121 & 96.6142116 & 3.385788404 & 121 & 23.55083038 & 76.44916962 \\
\hline 42 & 49.10008229 & 50.89991771 & 42 & 2.911117565 & 97.08888244 & 122 & 47463 & 3.249525368 & 122 & 34706 & 65294 \\
\hline 43 & 50.58924943 & 49.41075057 & 43 & 3.08 & 96.91385172 & 123 & 6307 & 3.115836931 & 123 & 5335 & 14665 \\
\hline 44 & 51.93 & 031 & 44 & 726 & 96.74 & 124 & 7008 & 915 & 124 & 5221 & 4779 \\
\hline 45 & 53.28237534 & 46.71762466 & 45 & 3.442246903 & 96.5577531 & 125 & 97.14629539 & 2.85370461 & 125 & 25.00118715 & 81285 \\
\hline 46 & 54.60 & 45.3 & 46 & 3.4 & 222 & 126 & 2941 & 589 & 126 & 5162 & 4838 \\
\hline 47 & 55.8 & 44.1 & 47 & 3.82 & 7773 & 127 & $97 .$. & 723 & 127 & 3485 & 5515 \\
\hline 48 & 57.13874323 & 42.86125677 & 48 & 4.010697037 & 95.98930296 & 128 & 97.49129166 & 2.508708343 & 128 & 26.16614961 & 85039 \\
\hline 49 & 58.38208384 & 91616 & 49 & 4.21 & 95.78 & 129 & 97.59961553 & 473 & 129 & 11366 & 8634 \\
\hline 50 & 59.60168093 & 1907 & 50 & 2894 & 16711 & 130 & 97.69725958 & 0422 & 130 & 26.97374873 & 25127 \\
\hline 51 & 60.75872479 & 39.24127521 & 51 & 4.611576176 & 95.38842382 & 131 & 97.79128012 & 2.208719881 & 131 & 6188 & 3812 \\
\hline 52 & 61.89803253 & 38.10196747 & 52 & 70538 & 95.16962946 & 132 & 97.88396568 & 2.116034317 & 132 & 27.81697442 & 02558 \\
\hline 53 & 63.0 & 069 & 53 & 5.0 & 8805 & 133 & 97. & 2.0 & 133 & 822 & $\overline{178}$ \\
\hline 54 & 64.07662007 & 37993 & 54 & 5.04 & 94.9 & 134 & 6382 & 5183 & 134 & 5054 & 4946 \\
\hline 55 & 65.12133513 & 34.87866487 & 55 & 5.472985017 & 94.52701498 & 135 & 98.13513212 & 1.86486788 & 135 & 29.14355692 & 544308 \\
\hline 56 & 66.18397703 & 33.81602297 & 56 & 5.695406464 & 59354 & 136 & 98.20922336 & 76642 & 136 & 6599 & 463401 \\
\hline 57 & 67.16 & 4719 & 57 & 5.7059 & 7022 & 137 & 560313 & 1.71 & 137 & 3699 & 06301 \\
\hline 58 & 68.14744252 & 31.85255748 & 58 & 6.160 & 93.83994485 & 138 & 98.35387768 & 232 & 138 & 30.5 & 3133 \\
\hline 59 & 69.11081932 & 30.88 & 59 & 6.160 & 93.8 & 139 & 8256 & 436 & 139 & 5762 & 14238 \\
\hline 60 & 70.0 & 508 & 60 & 6.6 & & 140 & & & 140 & & \\
\hline 61 & 70.93 & 99463 & 61 & 5461 & 93.36 & 141 & 8189 & 8106 & 141 & 3914 & 96086 \\
\hline 62 & 71.83922678 & 28.16077322 & 62 & 7.119514601 & 92.8804854 & 142 & 98.60237416 & 1.397625838 & 142 & 32.6348172 & 51828 \\
\hline 63 & 72.70133603 & 27.29866397 & 63 & 7.377156952 & & 143 & & 89592 & 143 & & 55622 \\
\hline 64 & $73.52 \mathrm{C}$ & 26.47078736 & 64 & 7.37 & 92. & 144 & 5248 & 521 & 144 & 573 & 427 \\
\hline 65 & 74.36366878 & 25.63633122 & 65 & 7.889 & 92.11011162 & 145 & 98.75236839 & 31607 & 145 & 34.30857378 & $\overline{42622}$ \\
\hline 66 & 75.144 & 6023 & 66 & 7.85 & 5824 & 146 & 9971 & 293 & 146 & 2116 & 47884 \\
\hline 67 & 75.9 & & 67 & & & 147 & & & 147 & & 5739 \\
\hline 68 & 76.6 & 23.3 & 68 & 8.6 & $\overline{009}$ & 148 & $\overline{214}$ & 786 & 148 & 36. & $\overline{088}$ \\
\hline 69 & 77.40427021 & 22.59572979 & 69 & 8.945608757 & 91.05439124 & 149 & 98.92658293 & 1.073417075 & 149 & 36.75446996 & 63.24553004 \\
\hline 70 & 78.07 & 2201 & 70 & 8.9 & 124 & 150 & 58333 & & 150 & 405 & 157595 \\
\hline 71 & 78.79522116 & 884 & 71 & 9.21 & 90. & 151 & 5094 & 059 & 151 & 921 & 7079 \\
\hline 72 & 79.44478296 & 704 & 72 & 9.4 & 6445 & 152 & 3713 & 872 & 152 & 4837 & 65163 \\
\hline 73 & 80.10397566 & 2434 & 73 & 9.7 & 3169 & 153 & 7194 & 3063 & 153 & 97674 & 02326 \\
\hline 74 & 80.7 & & 74 & & & 154 & & & 154 & & \\
\hline 75 & 81.3 & 4222 & 75 & 10.3 & 26487 & 155 & 99. & 175 & 155 & 4919 & 85081 \\
\hline 76 & 81.92574284 & 18.07425716 & 76 & 10.58514666 & 89.41485334 & 156 & 99.15877361 & 0.841226386 & 156 & 41.39570711 & 58.60429289 \\
\hline 77 & 82.51 & 3247 & 77 & 10.8 & $\overline{0942}$ & 157 & 9174 & & 157 & 42.09091432 & 57.90908568 \\
\hline 78 & 83.00 & & 78 & & & 158 & & & 158 & 8115 & 57.20201885 \\
\hline 79 & 91448 & 3552 & 79 & 3792 & 88.58716208 & 159 & 99.23114845 & 0.768851548 & 159 & 43.4 & 81386 \\
\hline 80 & 84.14418899 & 15.85581101 & 80 & 11.69639484 & 88.30360516 & 160 & 99.25260344 & 0.747396556 & 160 & 44.19056705 & 55.80943295 \\
\hline 81 & 84.67 & & 81 & & & 161 & & & 161 & & 71461 \\
\hline 82 & & 14.84466109 & 82 & & 3763 & 162 & 99.29 & 44348 & 162 & 45.63464802 & 35198 \\
\hline 83 & 85.65404827 & 14.34595173 & 83 & 12.50840851 & 87.49159149 & 163 & 99.30733751 & 0.692662488 & 163 & 46.35269155 & 53.64730845 \\
\hline 84 & 86.12596273 & 13.87403727 & 84 & 12.77709916 & 87.22290084 & 164 & 99.32631326 & 0.67368674 & 164 & 47.07684385 & 52.92315615 \\
\hline 85 & 86.5 & 438 & 85 & $\overline{036}$ & 9964 & 165 & 8868 & 1325 & 165 & 2425 & $\overline{77575}$ \\
\hline 86 & & & 86 & & & 166 & & & 166 & 6336 & 664 \\
\hline 87 & 87.4671381 & 12.5328619 & 87 & 13.63447525 & 86.36552475 & 167 & 99.37408637 & 0.625913625 & 167 & 49.27798338 & 50.72201662 \\
\hline 88 & 87.87220835 & 12.12779165 & 88 & 13.8 & 26885 & 168 & 99.38734079 & 9208 & 168 & 50.01642926 & 49.9 \\
\hline 89 & 88.2 & 11. & 89 & 14. & 85 & 169 & 7199 & 0.5 & 169 & $\overline{503}$ & 400497 \\
\hline 90 & 88.70542487 & 11.29457513 & 90 & 14.6765702 & 85.3234298 & 170 & 99.41203787 & 0.587962129 & 170 & 51.49826055 & 48.50 \\
\hline
\end{tabular}




\begin{tabular}{|c|c|c|c|c|c|c|c|c|c|c|c|}
\hline 171 & 99.42338518 & 0.576614822 & 171 & 52.24436627 & 47.75563373 & 251 & 99.605991 & 0.394009003 & 251 & 99.06442681 & 0.935573186 \\
\hline 172 & 99.4349232 & 0.565076804 & 172 & 52.97341036 & 47.02658964 & 252 & 99.605991 & 0.394009003 & 252 & 99.13479704 & 0.865202963 \\
\hline 173 & 99.44417268 & 0.555827319 & 173 & 53.71240508 & 46.28759492 & 253 & 99.605991 & 0.394009003 & 253 & 99.20712398 & 0.792876023 \\
\hline 174 & 99.45494785 & 0.545052145 & 174 & 54.4624481 & 45.5375519 & 254 & 99.605991 & 0.394009003 & 254 & 99.27945092 & 0.720549083 \\
\hline 175 & 99.4660091 & 0.533990905 & 175 & 55.21943507 & 44.78056493 & 255 & 99.605991 & 0.394009003 & 255 & 99.80280111 & 0.197198888 \\
\hline 176 & 99.47401896 & 0.525981041 & 176 & 55.96628052 & 44.03371948 & & & & & & \\
\hline 177 & 99.48174276 & 0.518257244 & 177 & 56.71047724 & 43.28952276 & \multirow{3}{*}{\multicolumn{3}{|c|}{$\begin{array}{l}\text { Reconstructed image using } \\
\text { pixel averaging formula } \\
(\text { Size }=2353 \times 1781)\end{array}$}} & \multirow{3}{*}{\multicolumn{3}{|c|}{$\begin{array}{l}\text { Reconstructed image using } \\
\text { extended morphological } \\
\text { filtering (Size }=2353 \times 1781)\end{array}$}} \\
\hline 178 & 99.49175509 & 0.508244915 & 178 & 57.47206966 & 42.52793034 & & & & & & \\
\hline 179 & 99.50014637 & 0.499853629 & 179 & 58.23869704 & 41.76130296 & & & & & & \\
\hline 180 & 99.50663055 & 0.493369454 & 180 & 58.99938268 & 41.00061732 & & & & & & \\
\hline 181 & 99.51301937 & 0.486980634 & 181 & 59.74899617 & 40.25100383 & Th & lity & & $\mathrm{Th}$ & Quality & \\
\hline 182 & 99.51759643 & 0.482403569 & 182 & 60.52798427 & 39.47201573 & 0 & $9.53555 \mathrm{E}-05$ & 99.99990464 & 0 & 0.229269956 & 99.77073004 \\
\hline 183 & 99.52 & 0.474 & 183 & 61.2 & 9401 & 1 & 0.001811755 & 99.99818825 & 1 & 0.255447011 & 74455299 \\
\hline 184 & 99.53142298 & 0.468577019 & 184 & 62.07884949 & 37.92115051 & 2 & 0.020882859 & 99.97911714 & 2 & 0.377407746 & 99.62259225 \\
\hline 185 & 99.53619076 & 0.463809243 & 185 & 62.88902575 & 37.11097425 & 3 & 0.141126168 & 99.85887383 & 3 & 0.724892041 & 99.27510796 \\
\hline 186 & 99.54 & 0.4585 & 186 & 63.7 & $\frac{36.29}{25.17}$ & 4 & 0.513584824 & 99.48641518 & 4 & 1.316202356 & 98.68379764 \\
\hline 187 & 99.54620309 & 0.453796914 & 187 & 64.52 & 35.47816554 & 5 & 1.277859307 & 4069 & 5 & 664 & 9336 \\
\hline 188 & 99.54954053 & 0.450459471 & 188 & 65.36038312 & 34.63961688 & 6 & 2.497742458 & 97.50225754 & 6 & 59521 & 993048 \\
\hline 189 & 99.55 & 0.446168472 & 189 & 66.20 & 33.79405268 & 7 & 4.190112205 & 95.8098878 & 7 & 5.197350414 & 94.80264959 \\
\hline 190 & 99.55 & 0.44 & 190 & 67.0 & $\frac{32.9}{320}$ & 8 & 6.262569049 & 93.73743095 & 8 & 7.132376435 & 92.86762356 \\
\hline 191 & 99.55783646 & 0.44216354 & 191 & 67.93 & $\frac{32.063}{31.187}$ & 9 & 8.699 & 748 & 9 & 9.31 & $\overline{508}$ \\
\hline 192 & 99.56136461 & 0.438635386 & 192 & 68.81277154 & $\frac{31.1872}{303339}$ & 10 & 11.38640249 & 88.61359751 & 10 & 4601 & 15399 \\
\hline 193 & 99.56 & 0.435 & 193 & 69.6 & $\frac{30.3}{20.4}$ & 11 & 14.28683131 & 85.71316869 & 11 & 14.18521949 & 85.81478051 \\
\hline$\frac{194}{195}$ & $\frac{99.5}{09.5}$ & $\frac{0.43}{0.42}$ & $\begin{array}{l}194 \\
105\end{array}$ & $\frac{70.5}{71.2}$ & 29.4 & 12 & 17.31255727 & 82.68744273 & 12 & 16.81010754 & 83.18989246 \\
\hline $\begin{array}{l}195 \\
196\end{array}$ & $\frac{99.57}{99.57}$ & $\frac{0.429}{0.425}$ & $\begin{array}{l}195 \\
196\end{array}$ & $\frac{71 . .}{72.3}$ & $\frac{62}{18}$ & 13 & 167 & & 13 & 19.5 & 4873 \\
\hline 197 & 99.57 & $0.420^{\circ}$ & 197 & 73.2 & 26.76 & 14 & 23.47 & 76.52556911 & 14 & 22.21 & 4183 \\
\hline 198 & 99.58 & 0.418 & 198 & 74.15 & 25.849 & 15 & 26.57 & 73.42851721 & 15 & 0273 & 75.0279727 \\
\hline 199 & 99.58262889 & 0.417371106 & 199 & 75.04 & 24.95704171 & 16 & 29.6 & 70. & 16 & & 6339 \\
\hline 200 & 99.58 & 0.413366174 & 200 & 75.96 & 24.03239751 & 17 & 32.7 & 67. & 17 & 618 & 382 \\
\hline 201 & 99.58 & 0.412221908 & 201 & 76.87 & 23.12884766 & 18 & 35.68 & 64.3 & 18 & 33.04 & 39822 \\
\hline 202 & 99.59006662 & 0.409933375 & 202 & 77.75 & 22.245 & 19 & 38.5 & 61.42783447 & 19 & 35.64610913 & 64.35389087 \\
\hline 203 & 99.59149696 & 0.408503042 & 203 & 78.63148171 & 21.36851829 & 20 & 0385 & 58.62409615 & 20 & 38.1 & 564776 \\
\hline 204 & 99.59 & 0.40707271 & 204 & 79.50 & 20.49 & 21 & 945 & 55. & 21 & 40.6 & 9482 \\
\hline 205 & 99.59550189 & 0.404498111 & 205 & 80.38 & 19.61 & 22 & 46.73 & 53.26912093 & 22 & 791 & 209 \\
\hline 206 & 99.59 & 0.403639911 & 206 & 81.23 & 18.76174179 & 23 & 67578 & 50.72732422 & 23 & 36138 & 54.62363862 \\
\hline 207 & 99.59 & 0.40 & 207 & 82.08 & 876 & 24 & 0624 & 48. & 24 & 47. & 9527209 \\
\hline 208 & 99.59845791 & 0.401542089 & 208 & 82.90 & 17.09593139 & 25 & 608 & 45.96374392 & 25 & 49.7 & 7978 \\
\hline 209 & 99.59903004 & 0.400969956 & 209 & 83.72066386 & 16.27933614 & 26 & 56.2 & 43.72718023 & 26 & 55619 & 48.13194381 \\
\hline 210 & 99.59969753 & 0.400302468 & 210 & 84.50 & 841 & 27 & 58.4 & & 27 & 53.8 & 49597 \\
\hline 211 & 99.600 & 0.39 & 211 & 85.29 & 477 & 28 & 7681 & & 28 & 552 & 0448 \\
\hline 212 & 99.60 & 0.399158201 & 212 & 86.03 & 13.9602209 & 29 & 159 & 37.5 & 29 & 0359 & 29641 \\
\hline 213 & 99.600 & 0.399062846 & 213 & 86.76 & 13.23093818 & 30 & 64.3 & 35. & 30 & 59.4 & 48865 \\
\hline 214 & 99.60 & 68 & 214 & 87.4 & 851 & 31 & & & 31 & 0371 & 39629 \\
\hline 215 & 99.60 & 0.3977 & 215 & 88.1 & 2606 & 32 & 127 & & 32 & 6073 & 3927 \\
\hline 216 & 99.60236749 & 0.397632513 & 216 & 88.85 & 11.14367003 & 33 & 9356 & 30. & 33 & 64.327 & 28618 \\
\hline 217 & 99.60 & 0.396869669 & 217 & 89.4 & & 34 & 70.9 & 29. & 34 & 65 & 34.12192208 \\
\hline 218 & 99.60 & 0.3 & 218 & 90.1 & 851 & 35 & & & 35 & & 85444 \\
\hline 219 & 99.60417924 & 758 & 219 & 065 & 4429346 & 36 & 895 & 26 & 36 & 68. & 3603 \\
\hline 220 & 99.60 & 0.39 & 220 & & 77799 & 37 & 5308 & 1692 & 37 & 70. & 8189 \\
\hline 221 & 99.60 & 59 & 221 & 91. & 88 & 38 & 395 & 23. & 38 & 29041 & 28.69670959 \\
\hline 222 & 99.60 & 426 & 222 & 985 & 149 & 39 & & & 39 & & \\
\hline 223 & 99.60 & 0.394 & 223 & 92.8 & 921 & 40 & 081 & 19 & 40 & 73. & 509 \\
\hline 224 & 99.6 & & 224 & & 06 & 41 & 9771 & 229 & 41 & 74.8 & 3686 \\
\hline 225 & 99.6 & 03 & 225 & 93.77 & 6.226 & 42 & 41248 & 58752 & 42 & 99695 & 24.12300305 \\
\hline 226 & 99.6 & 0.3 & 226 & 94.18 & 6252 & 43 & & & 43 & & \\
\hline 227 & 99. & 0.3 & 227 & 53 & 373 & 44 & 732 & 17 & 44 & 77. & 715 \\
\hline 228 & & & 228 & & & 45 & 83.80 & 055 & 45 & 78.8 & 962351 \\
\hline 229 & 99.6 & 03 & 229 & 95.2 & 795 & 46 & 757 & 243 & 46 & 79.73447351 & 20.26552649 \\
\hline 230 & 99.60 & 0.394009003 & 230 & 95.61 & 9714 & 47 & & & 47 & & 411 \\
\hline 231 & 99.6 & 0.3940 & 231 & 95.9 & & 48 & $86.1^{7}$ & & 48 & 277 & 723 \\
\hline 232 & 99.6 & 3 & 232 & 96.1 & 3.8 & 49 & 86.90349163 & 13.0 & 49 & 82.21 & 17.78572184 \\
\hline 233 & 99.6 & 003 & 233 & 96.4 & 3.55 & 50 & 87.5 & 352 & 50 & 9157 & 17.01250843 \\
\hline 234 & 99. & & 234 & 96. & & 51 & 112 & & 51 & & \\
\hline 235 & & & 235 & & & 52 & 3114 & & 52 & $\overline{58}$ & 15 \\
\hline 236 & 99.6 & 03 & 236 & 97.1 & 27269 & 53 & 89.4 & 10. & 53 & 85.06 & 91954 \\
\hline 237 & 99.60 & 0.394009003 & 237 & 97.16 & 2.830701271 & 54 & 8693 & & 54 & 2479 & 417521 \\
\hline 238 & 99.6 & 003 & 238 & 97.55 & & 55 & 9337 & & 55 & & \\
\hline 239 & $\overline{99.6}$ & & 239 & 97.66 & 2.3 & 56 & 6094 & & 56 & 86.9 & 59451 \\
\hline 240 & 99.60 & 0.394009003 & 240 & 97.73 & 2.265591872 & 57 & 91.48484753 & 8.515152469 & 57 & 87.46097602 & 12.53902398 \\
\hline 241 & 99.60 & 0.394009003 & 241 & 98.00 & 1.930468302 & 58 & 056 & & 58 & 87.99 & 03781 \\
\hline 242 & & 0.394009003 & 242 & 98.07 & 1.928726347 & 59 & 537 & & 59 & 88.5 & \\
\hline 243 & 99.6 & 0.394009003 & 243 & 98.33409415 & 1.665905854 & 60 & 4457 & 7.2 & 60 & 89.0 & 221537 \\
\hline 244 & 99.605991 & 0.394009003 & 244 & 98.37427843 & 1.625721569 & 61 & 93.16081613 & 6.839183871 & 61 & 89.47140724 & 10.52859276 \\
\hline 245 & 99.605991 & 0.394009003 & 245 & 98.57245568 & 1.427544323 & 62 & 93.5291745 & 6.470 & 62 & 89.93512529 & 487471 \\
\hline 246 & 99.60 & 003 & 246 & & 1028 & 63 & 1757 & & 63 & 90.3787512 & 9.621248801 \\
\hline 247 & & 0.394009003 & 247 & 98.68 & 1.319901028 & 64 & 94.20667546 & 5.793324542 & 64 & 90.78071336 & 9.219286643 \\
\hline 248 & 99.605991 & 0.394009003 & 248 & 98.77884159 & 1.22115841 & 65 & 94.53260062 & 5.467399378 & 65 & 91.18842635 & 8.811573647 \\
\hline 249 & 99.605991 & 0.394009003 & 249 & 98.88049542 & 1.119504578 & 66 & 94.83201695 & 5.16798305 & 66 & 91.56122866 & 8.438771344 \\
\hline 250 & 99.605991 & 0.394009003 & 250 & 98.97153048 & 1.028469516 & 67 & 95.12990759 & 4.870092409 & 67 & 91.92155092 & 8.078449078 \\
\hline
\end{tabular}




\begin{tabular}{|c|c|c|c|c|c|c|c|c|c|c|c|}
\hline 68 & & & 08 & & & 148 & & & & & \\
\hline 69 & 95.66151461 & 338485392 & 69 & 92.61573205 & 7.384267948 & 149 & 9.48193347 & 0.518066533 & & 99.52370646 & \\
\hline 70 & 95.9084854 & 91514598 & 70 & 2.92582396 & 7.074176037 & 150 & 99.48469878 & 0.515301223 & 150 & 99.52993455 & 0.470065452 \\
\hline 71 & 96.14048538 & 859514621 & 71 & .24407681 & 5.755923185 & 151 & 99.48822693 & 0.511773069 & 151 & .53513655 & 464863449 \\
\hline 72 & 96.35894487 & .641055128 & 72 & 3.53538902 & & 152 & 99.49309006 & 0.506909938 & 152 & & 460100600 \\
\hline 73 & 96.56643848 & 61519 & 73 & & & 153 & 99.49585537 & 0.504144628 & 153 & 9.54542125 & 0.454578753 \\
\hline 74 & 96.77212033 & 3.227879665 & 74 & 94.10097566 & 5.899024338 & 154 & 99.49871604 & 0.501283962 & 154 & 99.55081415 & .449185851 \\
\hline 75 & 96.9 & & 75 & & & 155 & & & 155 & & 068 \\
\hline 76 & 97.12636609 & 2.873633913 & 76 & 4.61427979 & 385720214 & 156 & 99.50462808 & 87192 & 156 & & 3853 \\
\hline 77 & 97.28923331 & 6687 & 77 & 4.85715131 & 5.142848689 & 157 & 99.5075841 & 5899 & 157 & 9.56513159 & 6841 \\
\hline 78 & 97.44075323 & 2.559 & 78 & 5.08241238 & 4.917587616 & 158 & 99.51025406 & 0.489745944 & 158 & 99.5693791 & 20902 \\
\hline 79 & 97.58 & 2.41 & 79 & & 3786 & 159 & 99.5 & & 159 & & \\
\hline 80 & 97.72 & 2.2 & 80 & 164 & & 160 & 99.51511719 & & 160 & & \\
\hline 81 & 97.85 & & 81 & & 4.276 & 161 & 99.51759643 & & 161 & & \\
\hline 82 & . & & 82 & & & 162 & & & 162 & & \\
\hline 83 & 98.0 & & 83 & & & 163 & & & 163 & & \\
\hline 84 & 98.19 & & 84 & 449 & 3.728 & 164 & 99.5 & 4417 & 164 & & 4454 \\
\hline 85 & 98.2 & & 85 & & & 165 & 789483 & & 165 & & 4406 \\
\hline 86 & 98.38 & & 86 & & & 166 & & & 166 & & \\
\hline 87 & 98.4 & 1.5 & 87 & & 3.2 & 167 & 99.5 & & 167 & & \\
\hline 88 & 98.54 & & 88 & & & 168 & 99.5 & & 168 & & 8757 \\
\hline 89 & 98.6 & & 89 & & & 169 & 99. & & 169 & & \\
\hline 90 & 986 & & 90 & & & 170 & 99. & & 170 & & \\
\hline 91 & 98.7 & & 91 & & & 171 & & & 171 & & \\
\hline 92 & 98.80 & & 92 & & & 172 & & & 172 & & 2188 \\
\hline 93 & 98.8 & & 93 & & 2.45 & 173 & 99. & & 173 & & \\
\hline 94 & 98.9 & & 94 & & & 174 & 99. & & 174 & & \\
\hline 95 & 98.95 & & 95 & & & 175 & & & 175 & & 072 \\
\hline 96 & 98.9 & & 86 & & & 17 & & & 176 & & \\
\hline 97 & 99.0 & & 91 & & & 177 & & & 177 & & \\
\hline 98 & 99.06 & & 98 & & & 178 & & & 178 & & \\
\hline 99 & 99.11 & & 99 & & & 179 & & & 179 & & \\
\hline 100 & 99.1 & & 100 & & & 180 & & & 180 & & \\
\hline 101 & 99.1 & & 101 & & & 181 & & & 181 & & \\
\hline 102 & 99.1 & & 102 & & & 182 & & & 182 & & \\
\hline 103 & 99.2 & & 103 & & & 18 & & & 18 & & \\
\hline 104 & 8 & & 104 & & & 18 & & & 10 & & \\
\hline 105 & 0028 & & 105 & & & 185 & & & 185 & & 727 \\
\hline 106 & 99.2 & & 106 & & & 186 & & & 186 & & \\
\hline 107 & 99.2 & & 107 & & & 18 & & & 18 & & \\
\hline 108 & 99.3 & & 108 & & & 18 & & & 18 & & \\
\hline 109 & 99.3 & & 109 & & & 18 & & & 18 & & \\
\hline 110 & 99. & & 111 & & & $\overline{19}$ & & & & & \\
\hline 111 & & & 11 & & & & & & & & \\
\hline 112 & 56 & & 112 & & & 192 & & & 192 & & 209 \\
\hline 113 & 99.3 & & 113 & & & 193 & & & 93 & & \\
\hline 114 & 99.3 & & 114 & & & 19 & & & 194 & & \\
\hline 115 & & & 11 & & & 19 & & & 195 & & \\
\hline 116 & & & 116 & & & 19 & & & 19 & & \\
\hline 117 & & & 11 & & & 19 & & & & & \\
\hline 118 & & & 11 & & & 19 & & & $\overline{19}$ & & \\
\hline 119 & & & 119 & & & 199 & & & 199 & & 109 \\
\hline 120 & & & 12 & & & 20 & & & 20 & & \\
\hline 121 & & & & & & 70 & & & & & \\
\hline 122 & 99.4 & & & & & 202 & & & 20 & & 168 \\
\hline 123 & & & 123 & & & 20 & & & 203 & & \\
\hline 124 & & & & & & & & & & & \\
\hline 125 & & & & & & 20 & & & & & \\
\hline 126 & & & 12 & & & 20 & & & 206 & 47 & 5297 \\
\hline 127 & & & 127 & & & 20 & & & 20 & & \\
\hline 128 & & & & & & & & & & & \\
\hline 129 & & & & & & & & & 209 & & \\
\hline 130 & & & 130 & & & 21 & & & 210 & & 672 \\
\hline 131 & & & & & & & & & & & \\
\hline 132 & & & & & & 21 & & & $\sqrt{512}+2$ & & 09 \\
\hline 133 & & & 13 & & & 21. & & & 213 & & 7813 \\
\hline 134 & & & & & & 21 & & & 214 & & \\
\hline 135 & & & & & & & & & & & \\
\hline 136 & 99.4 & & & & & & & & 21 & & 6607 \\
\hline 137 & 99. & & 137 & & & 217 & & & 217 & & 3541 \\
\hline 138 & & & & & & 21 & & & & & \\
\hline 139 & & & & & & & & & & & \\
\hline 140 & 99.4 & & 140 & & & 220 & 351 & 492 & 220 & 518 & 9482 \\
\hline 141 & 99.4 & & 14 & & & 221 & 99.6 & & 221 & & \\
\hline$\overline{142}$ & & & & & & & & & & & \\
\hline 143 & & & & & & & & & & & \\
\hline 144 & 99.46 & & 144 & 28 & 17 & 22 & & 03 & 224 & 729 & 2714 \\
\hline 145 & & & 14 & & & & & & & & \\
\hline $146+2 \div$ & & & & & & & & & & & \\
\hline 147 & 611678 & 0.52 & 147 & $\overline{93}$ & 71 & 227 & & $\overline{03}$ & $\overline{7}$ & 22 & 0.21941 \\
\hline
\end{tabular}




\begin{tabular}{cccccc}
\hline 228 & 99.605991 & 0.394009003 & 228 & 99.78163516 & 0.218364838 \\
\hline \hline 229 & 99.605991 & 0.394009003 & 229 & 99.7836396 & 0.216360397 \\
\hline \hline 230 & 99.605991 & 0.394009003 & 230 & 99.78480886 & 0.215191139 \\
\hline 231 & 99.605991 & 0.394009003 & 231 & 99.78614516 & 0.213854845 \\
\hline \hline 232 & 99.605991 & 0.394009003 & 232 & 99.78714738 & 0.212852624 \\
\hline 233 & 99.605991 & 0.394009003 & 233 & 99.78779166 & 0.212208339 \\
\hline \hline 234 & 99.605991 & 0.394009003 & 234 & 99.78855526 & 0.211444742 \\
\hline \hline 235 & 99.605991 & 0.394009003 & 235 & 99.78958134 & 0.210418659 \\
\hline \hline 236 & 99.605991 & 0.394009003 & 236 & 99.79072674 & 0.209273263 \\
\hline 237 & 99.605991 & 0.394009003 & 237 & 99.79079832 & 0.209201676 \\
\hline \hline 238 & 99.605991 & 0.394009003 & 238 & 99.79282663 & 0.207173372 \\
\hline \hline 239 & 99.605991 & 0.394009003 & 239 & 99.79301753 & 0.206982473 \\
\hline \hline 240 & 99.605991 & 0.394009003 & 240 & 99.7935425 & 0.2064575 \\
\hline \hline 241 & 99.605991 & 0.394009003 & 241 & 99.79509356 & 0.204906444 \\
\hline \hline 242 & 99.605991 & 0.394009003 & 242 & 99.79509356 & 0.204906444 \\
\hline 243 & 99.605991 & 0.394009003 & 243 & 99.7961435 & 0.203856498 \\
\hline \hline 244 & 99.605991 & 0.394009003 & 244 & 99.7961435 & 0.203856498 \\
\hline \hline 245 & 99.605991 & 0.394009003 & 245 & 99.79716959 & 0.202830415 \\
\hline \hline 246 & 99.605991 & 0.394009003 & 246 & 99.79757525 & 0.202424754 \\
\hline \hline 247 & 99.605991 & 0.394009003 & 247 & 99.79757525 & 0.202424754 \\
\hline \hline 248 & 99.605991 & 0.394009003 & 248 & 99.79895927 & 0.201040735 \\
\hline \hline 249 & 99.605991 & 0.394009003 & 249 & 99.79926948 & 0.200730524 \\
\hline \hline 250 & 99.605991 & 0.394009003 & 250 & 99.79926948 & 0.200730524 \\
\hline \hline 251 & 99.605991 & 0.394009003 & 251 & 99.79984217 & 0.200157826 \\
\hline \hline 252 & 99.605991 & 0.394009003 & 252 & 99.79984217 & 0.200157826 \\
\hline \hline 253 & 99.605991 & 0.394009003 & 253 & 99.8004626 & 0.199537403 \\
\hline \hline 254 & 99.605991 & 0.394009003 & 254 & 99.80084439 & 0.199155605 \\
\hline \hline 255 & 99.605991 & 0.394009003 & 255 & 99.80280111 & 0.197198888 \\
\hline & & & & &
\end{tabular}

Fig. 7 shows graphical presentation of the values of ToT, and HVQT of the original gold mine image of size $117 \times 891$, its zero diluted version of size $2352 \times 1781$, the reconstructed image by interpolant prediction using pixel averaging and the reconstructed image by interpolant prediction using extended morphological filtering.

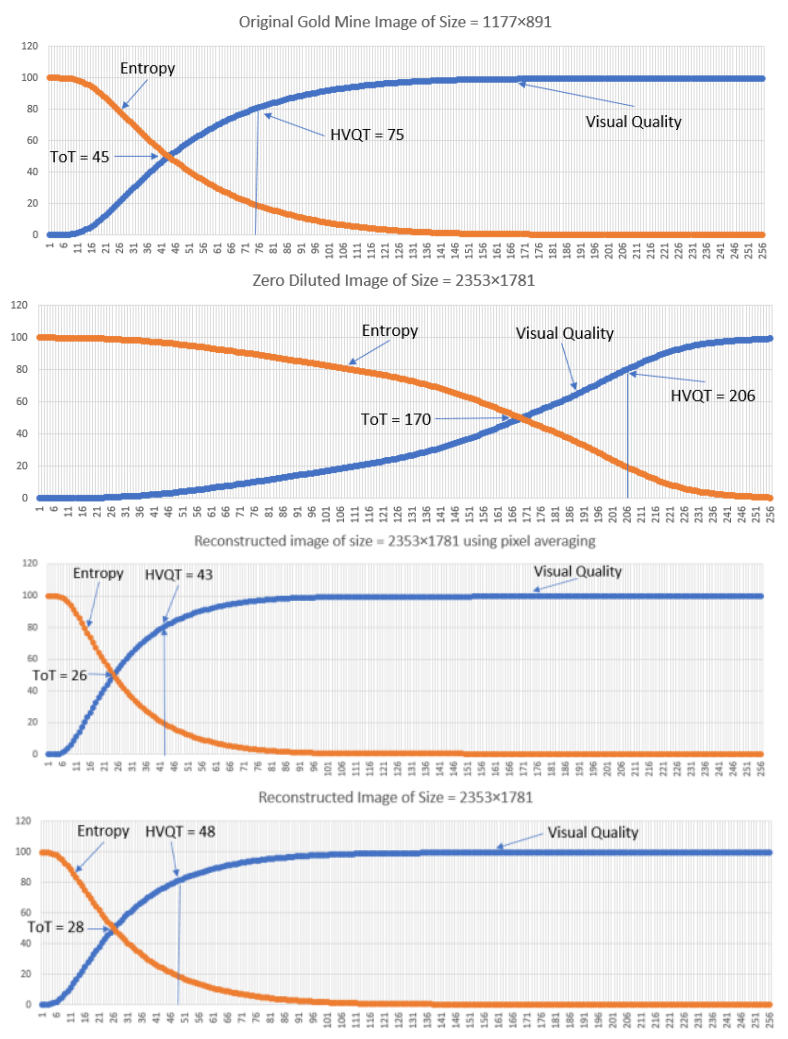

Fig. 7. Graphs of visual quality parametric values of sample image, zero diluted version and the reconstructed forms.

Observations

1. ToT and HVQT in zero diluted image are very poor (ie., ToT = 170 and HVQT = 206).
2. ToT and HVQT in the reconstructed image using prediction formula is pretty much closer to the original image.

3. ToT and HVQT in the reconstructed image using extended morphological filter is pretty much closer to the original image.

4. There is an increase in the contrast in the reconstructed images when compared to the original image.

\section{CONCLUSIONS}

Two methods for predicting interpolant pixel values of a zero diluted image (i) interpolant prediction using neighborhood pixel value averaging and (ii) interpolant prediction using extended morphological filtering are discussed briefly in this paper with a real time sample image. While both methods yield appreciable results, interpolant prediction using extended morphological filtering seems to be better than the interpolant prediction using neighborhood averaging, in the sense of prediction accuracy.

\section{ACKNOWLEDGEMENT}

The authors put on records the support extended by MGNIRSA, Hyderabad, Pentagram Research Centre Pvt Ltd., Hyderabad, University of Petroleum and Energy Studies, Dehradun and Avatar MedVision US LLC, NC, USA. Thanks are due to Dr.ShankarLingam, MG-NIRSA for coordinating with University of Mysore while the first author was carrying out $\mathrm{PhD}$.

Advisory Committee Members and Co-authors

1. Dr. Amit Agarwal, Director, APJ Abdul Kalam, Institute of Technology, Dehradun, India.

2. Dr. Michael Patrick Coyle, Chief Executive Officer, Avatar MedVision US LLC, NC, USA.

3. Sathya Govindarajan, Director, Pentagram Research Centre Private Limited, Hyderabad, India.

4. Prashanthi Govindarajan, Director, Pentagram Research Centre Private Limited, Hyderabad, India.

5. Yashaswi Vemuganti, Consultant, Avatar MedVision US LLC, NC, USA.

6. Dr. Jean Claude Perez, IBM European Research Center on Artificial Intelligence, France.

7. Dr. Hindupur Rajasimha, Chief Engineer, Indian Space Research Organization and IDBI (Retd.).

\section{REFERENCES}

[1] Jean Serra; Cube, cube-octahedron or rhomb-dodecahedron as bases for 3-D shape descriptions, Advances in visual form analysis, World Scientific 1997, 502-519.

[2] Wuthrich, C.A. and Stucki, P.; An algorithm comparison between square- and hexagonal based grids; Graphical Models and Image Processing, 53(4), 324-339,1991.

[3] Reinhard Klette and Azriel Rosenfeld; Digital Geometry: Geometric methods for picture analysis; Elsevier, 2004.

[4] B. Nagy; Geometry of Neighborhood sequences in hexagonal grid; Discrete Geometry of computer imagery; LNCS-4245, Springer.

[5] Tristan Roussillon, Laure Tougne, and Isabelle Sivignon; What Does Digital Straightness tell about Digital Convexity?.

[6] Edward Angel, "Interactive Computer Graphics- a top down approach with OpenGL", Second Edition, Addison Wesley, 2000.

[7] S.W. Zucker, R.A. Hummel, "A Three-Dimensional Edge Operator", IEEE Trans. On PAMI, Vol. 3, May 1981. 
[8] Jürgen, H., Manner, R., Knittel, G., and Strasser, W. (1995). Three Architectures for Volume Rendering. International Journal of the Eurographics Association, 14, 111-122.

[9] Dachille, F. (1997). Volume Visualization Algorithms and Architectures. Research Proficiency Examination, SUNY at Stony Brook.

[10] Günther, T., Poliwoda, C., Reinhart, C., Hesser, J., and Manner, J. (1994). VIRIM: A Massively Parallel Processor for Real-Time Volume Visualization in Medicine. Proceedings of the 9th Eurographics Hardware Workshop, 103-108.

[11] Boer, M.De, Gröpl, A., Hesser, J., and Manner, R. (1996). Latencyand Hazard-free Volume Memory Architecture for Direct Volume Rendering. Eurographics Workshop on Graphics Hardware, 109-119.

[12] Swan, J.E. (1997). Object Order Rendering of Discrete Objects. PhD. Thesis, Department of Computer and Information Science, The Ohio State University.

[13] Yagel, R. (1996). Classification and Survey of Algorithms for Volume Viewing. SIGGRAPH tutorial notes (course 34).

[14] Law, A. (1996). Exploiting Coherency in Parallel Algorithms for Volume Rendering. PhD. Thesis, Department of Computer and Information Science, The Ohio State University.

[15] Ray, H., Pfister, H., Silver, D., and Cook, T.A. (1999). Ray Casting Architectures for Volume Visualization. IEEE Transactions on Visualization and Computer Graphics, 5(3), 210-233.

[16] Yagel, R. (1996). Towards Real Time Volume Rendering. Proceedings of GRAPHICON' 96, 230-241.

[17] Kaufman, A.E. (1994). Voxels as a Computational Representation of Geometry. in The Computational Representation of Geometry SIGGRAPH '94 Course Notes.

[18] Lacroute, P., and Levoy, M. (1994). Fast Volume Rendering Using a Shear-Warp Factorization of the Viewing Transform. Computer Graphics Proceedings Annual Conference Series ACM SIGGRAPH, 451-458.

[19] Sutherland, I.E., Sproull, R.F., and Schumaker, R.A. (1974) A Characterization of Ten Hidden Surface Algorithms. ACM Computing Surveys, 6(1), 1-55.

[20] Roberts, J.C. (1993). An Overview of Rendering from Volume Data including Surface and Volume Rendering. Technical Report 13-93*, University of Kent, Computing Laboratory, Canterbury, UK.

[21] Frieder, G., Gordon, D., and Reynolds, R.A. (1985). Back-to-Front Display of Voxel-Based Objects. IEEE Computer Graphics and Applications, 5(1), 52-60.

[22] Westover, A.L. (1991). Splatting: A Parallel Feed-Forward Volume Rendering Algorithm. Ph.D. Dissertation, Department of Computer Science, The University of North Carolina at Chapel Hill.

[23] Zwicker, M., Pfister., H., Baar, J.B. and Gross M. (2001). Surface Splatting. In Computer Graphics SIGGRAPH 2001 Proceedings, 371-378.

[24] Nulkar, M., and Mueller, K. (2001). Splatting With Shadows. International Workshop on Volume Graphics 2001,35-50.

[25] J. Krüger and R. Westermann, Acceleration Techniques for GPUbased Volume Rendering, Proceedings of the 14th IEEE Visualization 2003 (VIS'03), 38-43.

[26] Markus Hadwiger, Joe M. Kniss, Christ of Rezk-salama, Daniel Weiskopf, and Klaus Engel. Real time Volume Graphics. A. K. Peters, Ltd., USA, 2006.

[27] Goodman, D., Nishimura, Y., Hongo, H and Noriaki N., 2006. Correcting for topography and the tilt of the GPR antenna, Archaeological Prospection, 13: 157-161.

[28] Goodman, D., Y. Nishimura, and J. D. Rogers, 1995. GPR time slices in archaeological prospection: Archaeological Prospection, 2:85-89.

[29] Goodman, D., 1994. Ground-penetrating radar simulation in engineering and archaeology: GEOPHYSICS, 59:224-232. 